\title{
A Note on Optimal Block-Scaling of Matrices
}

Dedicated to Prof. Dr. F.L. Bauer on the occasion of his 60 th birthday

\section{Elsner}

Fakultät für Mathematik, Universität Bielefeld, Postfach 8640, D-4800 Bielefeld (Fed. Rep.)

Summary. After pointing out that two recent results on optimal blockscaling are equivalent, a new short and simple proof of both results is given.

Subject Classifications: AMS(MOS): 65F35; CR: G1.3.

We use the following notation: $\rho$ is the spectralradius and \|\|$_{2}$ the spectralnorm, $\kappa_{2}(A)=\|A\|_{2}\left\|A^{-1}\right\|_{2}$ the condition of $A$ with respect to the spectralnorm and $C^{n, k}$ the set of all complex $n \times k$ matrices. Let $X_{1}, X_{2}$ be linear subspaces of $C^{n}, \operatorname{dim} X_{i}=n_{i}$ and $X_{1} \oplus X_{2}=C^{n}$. Let

$$
\mathscr{M}=\left\{A=\left(A_{1}, A_{2}\right): A_{i} \in C^{n, n_{\mathrm{i}}}, \operatorname{Im}\left(A_{i}\right)=X_{i}, i=1,2\right\} .
$$

Demmel [3] and Bart et al. [2] have proved

Theorem 1. Let $A=\left(A_{1}, A_{2}\right) \in \mathscr{M}$ and $A_{i}^{H} A_{i}=I_{n_{i}}$. Then

$$
\kappa_{2}(A)=\min \left\{\kappa_{2}(X), X \in \mathscr{M}\right\} .
$$

In other words, if the columns of $A_{i}$ are orthonormal bases of $X_{i}$ then $A$ has optimal condition.

Eisenstat et al. [4] have proved

Theorem 2. Let $B=\left(\begin{array}{cc}I_{n_{1}} & X \\ X^{H} & I_{n_{2}}\end{array}\right)$ be positive definite. Then

where

$$
\kappa_{2}(B)=\min \left\{\kappa_{2}\left(D^{H} B D\right): D \in \mathscr{D}\right\},
$$

$$
\mathscr{D}=\left\{D=D_{1} \oplus D_{2}: D_{i} \in C^{n_{i}, n_{i}} \text {, nonsingular }\right\} .
$$

By observing that $\left(\kappa_{2}(A)\right)^{2}=\kappa_{2}\left(A^{H} A\right)$ and that $C \in \mathscr{M} \Leftrightarrow C^{H} C=D^{H} B D$ for some $D \in \mathscr{D}$ and $B$ is a positive definite matrix of the form of Theorem 2, we see 
at once that Theorem 1 is an easy consequence of Theorem 2 . Similarly by taking Cholesky decomposition of $B$, Theorem 2 can be derived from Theorem 1 .

We give now a proof of Theorem 2 (and hence of Theorem 1) which is simpler than the proofs given before. The main tool is a trick used by F.L. Bauer in [1].

From $B\left(\begin{array}{l}x \\ y\end{array}\right)=\lambda\left(\begin{array}{l}x \\ y\end{array}\right)$ we get $X y=(\lambda-1) x, X^{H} x=(\lambda-1) y$ and $(\lambda-1)^{2} x$ $=X X^{H} x$. This shows that the eigenvalues of $B$ are given by $1 \pm \mu_{i}, \mu_{i}$ singular values of $X$. Also, if $z=\left(\begin{array}{l}x \\ y\end{array}\right)$ is an eigenvector belonging to the maximal eigenvalue $1+\|X\|_{2}$, then $\tilde{z}=\left(\begin{array}{r}x \\ -y\end{array}\right)=S z, S=I_{n_{1}} \oplus-I_{n_{2}}$ is an eigenvector belonging to the minimal eigenvalue $1-\|X\|_{2}$. This gives

$$
S B^{-1} S B z=\frac{1+\|X\|_{2}}{1-\|X\|_{2}} z=\kappa_{2}(B) \cdot z
$$

hence

$$
\rho\left(S B^{-1} S B\right) \geqq \kappa_{2}(B) .
$$

Now for any $D \in \mathscr{D}$, as $\|S\|_{2}=1$ and $S$ commutes with $D$,

$$
\begin{aligned}
\kappa_{2}(B) & \leqq \rho\left(S B^{-1} S B\right) \leqq\left\|D^{-1}\left(S B^{-1} S B\right) D\right\|_{2} \\
& =\left\|S\left(D^{-1} B^{-1} D^{-H}\right) S\left(D^{H} B D\right)\right\|_{2} \leqq \kappa_{2}\left(D^{H} B D\right) .
\end{aligned}
$$

\section{References}

1. Bauer, F.L.: Optimally scaled matrices. Numer. Math. 5, 73-87 (1963)

2. Bart, H., Gohberg, I., Kaashoek, M., Van Dooren, P.: Factorizations of transfer functions. SIAM J. Control Optimization 18, 675-696 (1980)

3. Demmel, J.: The condition number of equivalence transformations that block diagonalize matrix pencils. In: Matrix pencils (B. Kagström, A. Ruhe, eds.) Lecture Notes in Mathematics 973. Berlin, Heidelberg, New York: Springer 1982

4. Eisenstat, S.C., Lewis, J.W., Schultz, M.H.: Optimal block diagonal scaling of block 2-cyclic matrices. Linear Algebra Appl. 44, 181-186 (1982) 